AperTO - Archivio Istituzionale Open Access dell'Università di Torino

\title{
Numerical approximation of GB-splines by a convolutional approach
}

\section{This is the author's manuscript}

Original Citation:

Availability:

This version is available http://hdl.handle.net/2318/1621469

since 2017-01-11T03:33:23Z

Published version:

DOI:10.1016/j.apnum.2016.10.017

Terms of use:

Open Access

Anyone can freely access the full text of works made available as "Open Access". Works made available under a Creative Commons license can be used according to the terms and conditions of said license. Use of all other works requires consent of the right holder (author or publisher) if not exempted from copyright protection by the applicable law. 


\title{
Numerical approximation of GB-splines by a convolutional approach
}

\author{
Fabio Roman ${ }^{\mathrm{a}}$, Carla Manni ${ }^{\mathrm{b}}$, Hendrik Speleers $^{\mathrm{b}}$ \\ ${ }^{a}$ Department of Mathematics, University of Turin, Italy \\ ${ }^{b}$ Department of Mathematics, University of Rome 'Tor Vergata', Italy
}

\begin{abstract}
Generalized splines are smooth functions belonging piecewisely to spaces which are a natural generalization of algebraic polynomials. GB-splines are a B-spline-like basis for generalized splines, and they are usually defined by means of an integral recurrence relation which makes their evaluation quite cumbersome and computationally expensive. We present a simple strategy for approximating the values of a cardinal GB-spline of arbitrary degree $p$, with a particular focus on hyperbolic and trigonometric GB-splines due to their interest in applications. The proposed strategy is based on the Fourier properties of cardinal GB-splines. The approximant is expressed as a linear combination of scaled and dilated versions of (polynomial) cardinal B-splines of degree $p$, whose coefficients can be efficiently computed via discrete convolution. Sharp error estimates are provided and illustrated with some numerical examples.
\end{abstract}

Keywords: GB-splines, cardinal B-splines, convolution, Fourier transform

\section{Introduction}

Classical polynomial splines are smooth functions belonging piecewisely to the space of algebraic polynomials (of degree $p$ ). Generalized splines are smooth functions belonging piecewisely to spaces of the form

$$
\left\langle 1, x, \ldots, x^{p-2}, U(x), V(x)\right\rangle, \quad 2 \leq p \in \mathbb{N},
$$

where the functions $U, V$ are such that (1.1) is an extended Tchebycheff space; see [8]. Extended Tchebycheff spaces are natural generalizations of algebraic polynomial spaces. They are commonly used in approximation theory, mainly because they form a very flexible substitute for algebraic polynomial spaces to solve Hermite interpolation problems. Generalized splines are a special class of Tchebycheffian splines and possess all the desirable properties of polynomial splines. In particular, they admit a representation in terms of basis functions which are a natural generalization of the polynomial B-splines, the so-called generalized B-splines (GB-splines). Furthermore, the elegant and powerful blossoming approach and classical algorithms (like degree elevation, knot insertion, differentiation formulas, etc.) can be rephrased for them; see $[16,18]$. Interesting examples are trigonometric or hyperbolic (exponential) generalized splines for which $U, V$ are taken as $\cos (\alpha x), \sin (\alpha x)$, or $\cosh (\alpha x), \sinh (\alpha x)$, respectively.

Generalized splines and their B-spline-like basis are popular tools in the CAGD community; see, e.g., $[11,13,16,19]$. With a suitable choice of the functions $U, V$, they allow for an exact representation of conic sections as well as some transcendental curves (helix, cycloid, etc.). Moreover, tensor-product GB-splines are an interesting problem-dependent alternative to tensor-product polynomial B-splines and NURBS in isogeometric analysis, a successful paradigm for the analysis of problems governed by partial differential equations (see $[9,14,15]$ ). Their success and the need of local refinement motivated the recent study of generalized splines over T-meshes (see $[3,4,5,6]$ ).

Email addresses: fabio.roman@unito.it (Fabio Roman), manni@mat.uniroma2.it (Carla Manni), speleers@mat.uniroma2.it (Hendrik Speleers) 
GB-splines are usually defined by means of an integral recurrence relation. Using this recurrence relation for the evaluation of GB-splines, however, is quite cumbersome, computationally expensive, and numerically instable. This inherent complexity explains the often reluctant attitude to use GBsplines in practical applications, despite their interesting analytical and geometrical properties. In this paper we address the problem of a stable computation of values of cardinal GB-splines, which are GB-splines defined over a uniform knot sequence and are of particular interest in practical applications.

We present a simple strategy for approximating the values of a cardinal GB-spline of arbitrary degree $p$. The strategy can be applied to spaces of general $U, V$, and is illustrated in more detail for hyperbolic and trigonometric GB-splines due to their interest in applications. The proposed method exploits the Fourier properties of cardinal GB-splines [17] and the well-known refinement relation of (polynomial) cardinal B-splines [7]. The approximant is expressed as a linear combination of scaled and dilated versions of (polynomial) cardinal B-splines whose coefficients can be efficiently computed via discrete convolution. The accuracy of the approximation can be predetermined according to the application we need to deal with. Other approaches for the evaluation of GB-splines of low degree and particular selections of the functions $U, V$ can be found in [2].

The remainder of the paper is divided into four sections. Section 2 contains the definition of cardinal GB-splines and collects several of their properties. These properties are then exploited in the approximation strategy described in Section 3. In Section 4 we study the approximation error and give some numerical tests. Section 5 concludes the paper with some final remarks.

\section{Cardinal GB-splines}

In this section we discuss the definition and some properties of cardinal GB-splines. We focus on the space

$$
\mathbb{P}_{p}^{U, V}:=\left\langle 1, x, \ldots, x^{p-2}, U(x), V(x)\right\rangle, \quad x \in[0,1],
$$

where $U, V \in C^{p-1}[0, p+1]$ are such that $\left\{U^{(p-1)}, V^{(p-1)}\right\}$ is a Tchebycheff system on $[0,1]$, i.e., any non-trivial element in the space $\left\langle U^{(p-1)}, V^{(p-1)}\right\rangle$ has at most one zero in $[0,1]$. The space in $(2.1)$ will be called section space. We denote by $\widetilde{U}, \widetilde{V}$ the unique elements in the space $\left\langle U^{(p-1)}, V^{(p-1)}\right\rangle$ satisfying

$$
\widetilde{U}(0)=1, \tilde{U}(1)=0, \quad \widetilde{V}(0)=0, \tilde{V}(1)=1 .
$$

Popular examples of such a space (2.1) are

$$
\begin{aligned}
\mathbb{P}_{p} & :=\left\langle 1, x, \ldots, x^{p-2}, x^{p-1}, x^{p}\right\rangle, \\
\mathbb{H}_{p, \alpha}: & =\left\langle 1, x, \ldots, x^{p-2}, \cosh (\alpha x), \sinh (\alpha x)\right\rangle, \quad \alpha \in \mathbb{R}_{+}, \\
\mathbb{T}_{p, \alpha}: & =\left\langle 1, x, \ldots, x^{p-2}, \cos (\alpha x), \sin (\alpha x)\right\rangle, \quad 0<\alpha<\pi .
\end{aligned}
$$

Definition 2.1. The (normalized) cardinal GB-spline of degree $p \geq 1$ over the uniform knot set $\{0,1, \ldots, p+1\}$ with sections in $(2.1)$ is denoted by $\phi_{p}^{U, V}$ and is defined recursively as follows. For $p=1$,

$$
\phi_{1}^{U, V}(x):=\delta_{1}^{U, V} \begin{cases}\widetilde{V}(x), & \text { if } x \in[0,1), \\ \widetilde{U}(x-1), & \text { if } x \in[1,2), \\ 0, & \text { elsewhere, }\end{cases}
$$

where $\delta_{1}^{U, V}$ is a normalization factor given by

$$
\delta_{1}^{U, V}:=\left(\int_{0}^{1} \widetilde{V}(y) \mathrm{d} y+\int_{1}^{2} \widetilde{U}(y-1) \mathrm{d} y\right)^{-1} .
$$

For $p \geq 2$,

$$
\phi_{p}^{U, V}(x):=\int_{0}^{x}\left(\phi_{p-1}^{U, V}(y)-\phi_{p-1}^{U, V}(y-1)\right) \mathrm{d} y
$$


If the space (2.1) is the space of algebraic polynomials $\mathbb{P}_{p}$, then the function in Definition 2.1 is the classical (polynomial) cardinal B-spline of degree $p$, denoted by $\phi_{p}$, which can also be defined recursively as follows $[1]$ :

$$
\phi_{0}(x):= \begin{cases}1, & \text { if } x \in[0,1) \\ 0, & \text { elsewhere }\end{cases}
$$

and

$$
\phi_{p}(x):=\frac{x}{p} \phi_{p-1}(x)+\frac{p+1-x}{p} \phi_{p-1}(x-1), \quad p \geq 1 .
$$

The cardinal GB-spline $\phi_{p}^{U, V}$ is globally of class $C^{p-1}$ and possesses the following fundamental properties (see, e.g., [17, Section 3.1] and references therein). These properties are generalizations of well-known properties of $\phi_{p}$ (see, e.g., [10, Section 3.1]).

- Positivity:

$$
\phi_{p}^{U, V}(x)>0, \quad x \in(0, p+1)
$$

- Minimal support:

$$
\phi_{p}^{U, V}(x)=0, \quad x \notin(0, p+1) .
$$

- Partition of unity:

$$
\sum_{k \in \mathbb{Z}} \phi_{p}^{U, V}(x-k)=\sum_{k=1}^{p} \phi_{p}^{U, V}(k)=1, \quad p \geq 2 .
$$

- Recurrence relation for derivatives:

$$
\left(\phi_{p}^{U, V}\right)^{(r)}(x)=\left(\phi_{p-1}^{U, V}\right)^{(r-1)}(x)-\left(\phi_{p-1}^{U, V}\right)^{(r-1)}(x-1), \quad 1 \leq r \leq p-1 .
$$

- Conditional symmetry with respect to $\frac{p+1}{2}$ :

$$
\phi_{p}^{U, V}\left(\frac{p+1}{2}+x\right)=\phi_{p}^{U, V}\left(\frac{p+1}{2}-x\right) \quad \text { if } \quad \phi_{1}^{U, V}(1+x)=\phi_{1}^{U, V}(1-x) .
$$

- Convolution relation:

$$
\phi_{p}^{U, V}(x)=\left(\phi_{p-1}^{U, V} * \phi_{0}\right)(x):=\int_{\mathbb{R}} \phi_{p-1}^{U, V}(x-y) \phi_{0}(y) \mathrm{d} y=\int_{0}^{1} \phi_{p-1}^{U, V}(x-y) \mathrm{d} y, \quad p \geq 2,
$$

where $\phi_{0}(x):=\chi_{[0,1)}(x)$. Moreover,

$$
\phi_{p}^{U, V}(x)=(\phi_{1}^{U, V} * \underbrace{\phi_{0} * \cdots * \phi_{0}}_{p-1})(x), \quad \phi_{p}(x)=(\underbrace{\phi_{0} * \cdots * \phi_{0}}_{p+1})(x), \quad p \geq 1 .
$$

- Inner products:

$$
\int_{\mathbb{R}} \phi_{p_{1}}^{U_{1}, V_{1}}(x) \phi_{p_{2}}^{U_{2}, V_{2}}(x+k) \mathrm{d} x=(\phi_{1}^{U_{1}, V_{1}} * \phi_{1}^{U_{2}, V_{2}} * \underbrace{\phi_{0} * \cdots * \phi_{0}}_{p_{1}+p_{2}-2})\left(p_{2}+1-k\right),
$$

if $\phi_{1}^{U_{2}, V_{2}}(1+x)=\phi_{1}^{U_{2}, V_{2}}(1-x)$. In particular,

$$
\begin{gathered}
\int_{\mathbb{R}} \phi_{p_{1}}^{U, V}(x) \phi_{p_{2}}(x+k) \mathrm{d} x=(\phi_{1}^{U, V} * \underbrace{\phi_{0} * \cdots * \phi_{0}}_{p_{1}+p_{2}})\left(p_{2}+1-k\right)=\phi_{p_{1}+p_{2}+1}^{U, V}\left(p_{2}+1-k\right), \\
\int_{\mathbb{R}} \phi_{p_{1}}(x) \phi_{p_{2}}(x+k) \mathrm{d} x=(\underbrace{\phi_{0} * \cdots * \phi_{0}}_{p_{1}+p_{2}+2})\left(p_{2}+1-k\right)=\phi_{p_{1}+p_{2}+1}\left(p_{2}+1-k\right) .
\end{gathered}
$$




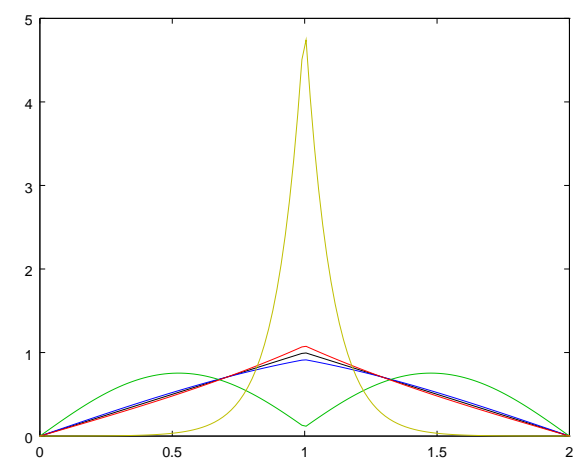

(a) $p=1$

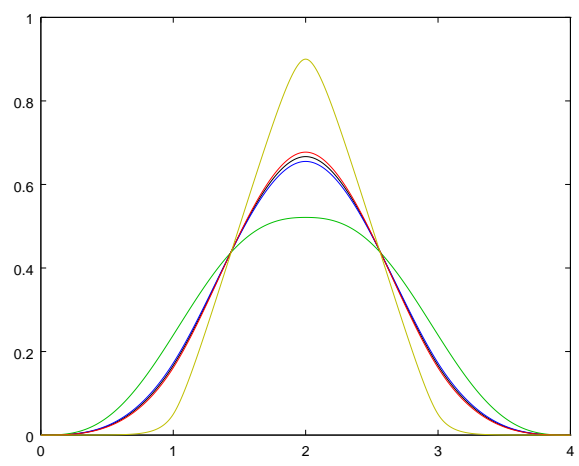

(b) $p=3$

Figure 1: Cardinal GB-splines of degree $p=1,3$ : polynomial B-spline (black), trigonometric GB-spline with $\alpha=1$ (blue) and $\alpha=3$ (green), hyperbolic GB-spline with $\alpha=1$ (red) and $\alpha=10$ (yellow).

- Unity of integral:

$$
\int_{0}^{p+1} \phi_{p}^{U, V}(x) \mathrm{d} x=1
$$

- Fourier transform:

$$
\widehat{\phi}_{p}^{U, V}(\theta)=\widehat{\phi}_{1}^{U, V}(\theta)\left(\frac{1-\mathrm{e}^{-\mathrm{i} \theta}}{\mathrm{i} \theta}\right)^{p-1} .
$$

We now give two examples of interest in practice: hyperbolic and trigonometric cardinal GBsplines with real phase parameters $\alpha$.

Example 2.1. The hyperbolic cardinal GB-spline is denoted by $\phi_{p}^{\mathbb{H}_{\alpha}}$ and is defined by taking $U(x):=$ $\cosh (\alpha x)$ and $V(x):=\sinh (\alpha x)$. In this case, we have

$$
\widetilde{U}(x)=\frac{\sinh (\alpha(1-x))}{\sinh (\alpha)}, \quad \widetilde{V}(x)=\frac{\sinh (\alpha x)}{\sinh (\alpha)},
$$

satisfying (2.2).

Example 2.2. The trigonometric cardinal GB-spline is denoted by $\phi_{p}^{\mathbb{T}_{\alpha}}$ and is defined by taking $U(x):=\cos (\alpha x)$ and $V(x):=\sin (\alpha x)$. In this case, we have

$$
\widetilde{U}(x)=\frac{\sin (\alpha(1-x))}{\sin (\alpha)}, \quad \widetilde{V}(x)=\frac{\sin (\alpha x)}{\sin (\alpha)},
$$

satisfying (2.2).

Note that $\phi_{p}^{\mathbb{H}_{\alpha}}=\phi_{p}^{\mathbb{T}_{\mathrm{i} \alpha}}$ with i the imaginary unit. To simplify the notation later on, we will also use the notation $\phi_{p}^{\mathbb{Q}_{\alpha}}$ if a statement holds for both $\phi_{p}^{\mathbb{H}_{\alpha}}$ and $\phi_{p}^{\mathbb{T}_{\alpha}}$. Some hyperbolic and trigonometric cardinal GB-splines are depicted in Figure 1.

Remark 2.1. Hyperbolic and trigonometric cardinal GB-splines approach the (polynomial) cardinal $B$-spline of the same degree as the phase parameter $\alpha$ approaches 0 , i.e.,

$$
\lim _{\alpha \rightarrow 0} \phi_{p}^{\mathbb{Q}_{\alpha}}(x)=\phi_{p}(x), \quad \mathbb{Q}=\mathbb{H}, \mathbb{T} .
$$

Remark 2.2. The symmetry requirement $\phi_{1}^{U, V}(1+x)=\phi_{1}^{U, V}(1-x)$ in (2.5) is equivalent to

$$
\widetilde{V}(x)=\widetilde{U}(1-x), \quad x \in[0,1] .
$$

This requirement is satisfied for hyperbolic and trigonometric cardinal GB-splines, see (2.8)-(2.9), as well as for (polynomial) cardinal B-splines. 
Remark 2.3. GB-splines can be defined in a more general setting than Definition 2.1. In particular, completely general knot sets can be considered and the section space (2.1) can be chosen differently on each knot interval; see, e.g., [14].

\section{Approximation strategy}

The integral recurrence relation in Definition 2.1 is not well suited for an efficient and numerically stable evaluation of cardinal GB-splines, due to both the need of integration and subtraction in the expression (2.3). Recurrence relations for Tchebycheffian splines and so for (cardinal) GB-splines analogous to (2.4) are also known; see [12]. However, they require quite involved weight functions and so they are more of theoretical interest than of practical help for designing good (numerical) evaluation algorithms. On the other hand, there exist extremely stable and fast algorithms for the evaluation of (polynomial) cardinal B-splines. In this section, we propose an approximation strategy for $\phi_{p}^{U, V}$ based on convolution of cardinal B-splines. The accuracy of the approximation can be predetermined according to the application we need to deal with.

Suppose we are given an approximation of the type:

$$
\phi_{1}^{U, V}(x) \simeq \sum_{k} q_{k}^{U, V} f_{k}(x) .
$$

The Fourier transform of (3.1) results in

$$
\widehat{\phi}_{1}^{U, V}(\theta) \simeq \sum_{k} q_{k}^{U, V} \widehat{f}_{k}(\theta),
$$

and can be extended to any $p \geq 2$ by means of $(2.7)$ :

$$
\widehat{\phi}_{p}^{U, V}(\theta) \simeq\left(\frac{1-\mathrm{e}^{-\mathrm{i} \theta}}{\mathrm{i} \theta}\right)^{p-1} \widehat{\phi}_{1}^{U, V}(\theta)=\sum_{k} q_{k}^{U, V} \widehat{f}_{k}(\theta) \widehat{\phi}_{p-2}(\theta) .
$$

Then, by taking the inverse Fourier transform of (3.2) we get

$$
\phi_{p}^{U, V}(x) \simeq \sum_{k} q_{k}^{U, V}\left(f_{k} * \phi_{p-2}\right)(x) .
$$

We now look for an intelligent choice of the functions $f_{k}$ and the coefficients $q_{k}^{U, V}$ in (3.1)-(3.3). For a fixed accuracy parameter $j$, linear spline interpolation over $\mathbb{Z} / 2^{j}$ gives

$$
\phi_{1}^{U, V}(x) \simeq \widetilde{\phi}_{1, j}^{U, V}(x):=\sum_{k=0}^{n_{j}} q_{k, j}^{U, V} \phi_{1}\left(2^{j} x-k\right),
$$

with $n_{j}:=2\left(2^{j}-1\right)$ and

$$
q_{k, j}^{U, V}:=\phi_{1}^{U, V}\left(\frac{k+1}{2^{j}}\right), \quad k=0, \ldots, n_{j} .
$$

When following the above procedure, we get for $p \geq 2$,

$$
\phi_{p}^{U, V}(x) \simeq \widetilde{\phi}_{p, j}^{U, V}(x):=\sum_{k=0}^{n_{j}} q_{k, j}^{U, V}\left(\phi_{1}\left(2^{j} \cdot-k\right) * \phi_{p-2}\right)(x) .
$$

In the convolution operation used in (3.6) we see that $\phi_{1}$ has as argument $2^{j} x-k$ while $\phi_{p-2}$ has as argument $x$. In order to deal with this, we recall the standard two-scale refinement formula for cardinal B-splines [7]:

$$
\phi_{p}(x)=\frac{1}{2^{p}} \sum_{i=0}^{p+1}\left(\begin{array}{c}
p+1 \\
i
\end{array}\right) \phi_{p}(2 x-i) .
$$

Iterating this recursively leads to the following refinement formula. 
Lemma 3.1. For every $p \geq 0$ and $j \geq 0$, we have

$$
\phi_{p}(x)=\sum_{l=0}^{N_{p, j}} a_{l, p, j} \phi_{p}\left(2^{j} x-l\right)
$$

with $N_{p, j}:=(p+1)\left(2^{j}-1\right), a_{0, p, 0}:=1$, and

$$
a_{l, p, m}:=\frac{1}{2^{p}} \sum_{k=\max \left(0,\left\lceil\frac{l-p-1}{2}\right\rceil\right)}^{\min \left(\left\lfloor\frac{l}{2}\right\rfloor, N_{p, m-1}\right)}\left(\begin{array}{c}
p+1 \\
l-2 k
\end{array}\right) a_{k, p, m-1}, \quad l=0, \ldots, N_{p, m}, \quad m>0 .
$$

Proof. The identity in (3.8) is trivial for $j=0$. We proceed by induction. Suppose the identity is true for $j-1 \geq 0$. Then, by means of (3.7) we have

$$
\phi_{p}(x)=\sum_{k=0}^{N_{p, j-1}} a_{k, p, j-1} \phi_{p}\left(2^{j-1} x-k\right)=\frac{1}{2^{p}} \sum_{k=0}^{N_{p, j-1}} a_{k, p, j-1} \sum_{i=0}^{p+1}\left(\begin{array}{c}
p+1 \\
i
\end{array}\right) \phi_{p}\left(2^{j} x-2 k-i\right) .
$$

By taking $l:=2 k+i$ and reorganizing the sums, we get (3.8). Note that

$$
2 N_{p, j-1}+p+1=(p+1)\left(2^{j}-2\right)+p+1=N_{p, j} .
$$

We now arrive at our main approximation result based on the procedure described in (3.1)-(3.3).

Theorem 3.1. For every $p \geq 2$ and $j \geq 0$, we have

$$
\phi_{p}^{U, V}(x) \simeq \widetilde{\phi}_{p, j}^{U, V}(x)=\sum_{r=0}^{N_{p, j}} b_{r, p, j}^{U, V} \phi_{p}\left(2^{j} x-r\right),
$$

with

$$
b_{r, p, j}^{U, V}:=\frac{1}{2^{j}} \sum_{k=\max \left(0, r-N_{p-2, j}\right)}^{\min \left(r, n_{j}\right)} q_{k, j}^{U, V} a_{r-k, p-2, j}, \quad r=0, \ldots, N_{p, j},
$$

and $q_{k, j}^{U, V}$ is defined in (3.5) and $a_{l, p, j}$ is defined in (3.9).

Proof. Using Lemma 3.1 we can rewrite (3.6) into

$$
\widetilde{\phi}_{p, j}^{U, V}(x)=\sum_{k=0}^{n_{j}} q_{k, j}^{U, V}\left(\phi_{1}\left(2^{j} \cdot-k\right) * \sum_{l=0}^{N_{p-2, j}} a_{l, p-2, j} \phi_{p-2}\left(2^{j} \cdot-l\right)\right)(x) .
$$

Moreover, from the translation and scaling properties of the convolution operator we know that

$$
\left(\phi_{1}\left(2^{j} \cdot-k\right) * \phi_{p-2}\left(2^{j} \cdot-l\right)\right)(x)=\frac{1}{2^{j}} \phi_{p}\left(2^{j} x-k-l\right),
$$

and we get

$$
\widetilde{\phi}_{p, j}^{U, V}(x)=\sum_{k=0}^{n_{j}} q_{k, j}^{U, V} \sum_{l=0}^{N_{p-2, j}} \frac{a_{l, p-2, j}}{2^{j}} \phi_{p}\left(2^{j} x-k-l\right) .
$$

By taking $r:=k+l$ and reorganizing the sums, we obtain (3.10). Note that $n_{j}+N_{p-2, j}=N_{p, j}$.

Remark 3.1. The expression for the coefficients given in (3.11) is a discrete convolution and therefore can be implemented efficiently. 
Remark 3.2. Thanks to the structure of the approximation in (3.10) and the expression of the coefficients (3.11), a more accurate solution $\widetilde{\phi}_{p, j+1}^{U, V}$ can be efficiently obtained from $\widetilde{\phi}_{p, j}^{U, V}$ by saving all the computations previously done both for the coefficients $a_{l, p, j}$ and for the values $q_{k, j}^{U, V}$.

Lemma 3.1 gives us a recursive procedure to compute the coefficients $a_{l, p, j}$. Inspired by a direct check for several values of $p$ and $j$, we conjecture the following explicit expression for the coefficients $a_{l, p, j}$ in (3.8):

$$
a_{l, p, j}=\frac{1}{2^{p j}} \sum_{k=0}^{\min \left(p+1,\left\lfloor\frac{l}{2^{j}}\right\rfloor\right)}(-1)^{k}\left(\begin{array}{c}
p+1 \\
k
\end{array}\right)\left(\begin{array}{c}
p+l-k 2^{j} \\
p
\end{array}\right) .
$$

This means that the coefficients $a_{l, p, j}$ can be written in terms of combinatorics as

$$
a_{l, p, j}=\frac{1}{2^{p j}} P\left(l, p+1,2^{j}-1\right),
$$

where $P(\alpha, \beta, \gamma)$ stands for the number of ways that the nonnegative integer $\alpha$ can be written as an ordered sum of $\beta$ nonnegative integers ranging from 0 to $\gamma$; see [20].

Under the validity of the above conjecture, we can also formulate an alternative expression for the coefficients in (3.10). Combining (3.11) and (3.12) gives

$$
b_{r, p, j}^{U, V}=\frac{1}{2^{(p-1) j}} \sum_{k=\max \left(0, r-N_{p-2, j}\right)}^{\min \left(r, n_{j}\right)} q_{k, j}^{U, V} \sum_{l=0}^{\min \left(p-1,\left\lfloor\frac{r-k}{2^{j}}\right\rfloor\right)}(-1)^{l}\left(\begin{array}{c}
p-1 \\
l
\end{array}\right)\left(\begin{array}{c}
p-2+r-k-l 2^{j} \\
p-2
\end{array}\right) .
$$

In the explicit expression (3.13) of the coefficients $b_{r, p, j}^{U, V}$ we only need to know the function values of $\phi_{1}^{U, V}$ in integer multiples of $\frac{1}{2^{j}}$ between 0 and 2 ; see (3.5).

Example 3.1. The function values of $\phi_{1}^{\mathbb{H}_{\alpha}}$ in multiples of $\frac{1}{2^{j}}$ between 0 and 2 are given by

$$
\phi_{1}^{\mathbb{H}_{\alpha}}\left(\frac{k+1}{2^{j}}\right)= \begin{cases}\frac{\alpha}{2 \tanh (\alpha / 2)} \frac{\sinh \left(\frac{k+1}{2^{j}} \alpha\right)}{\sinh (\alpha)}, & \text { if } k \leq 2^{j}-1, \\ \frac{\alpha}{2 \tanh (\alpha / 2)} \frac{\sinh \left(\left(2-\frac{k+1}{2^{j}}\right) \alpha\right)}{\sinh (\alpha)}, & \text { if } k \geq 2^{j}-1 .\end{cases}
$$

Example 3.2. The function values of $\phi_{1}^{\mathbb{T}_{\alpha}}$ in multiples of $\frac{1}{2^{j}}$ between 0 and 2 are given by

$$
\phi_{1}^{\mathbb{T}_{\alpha}}\left(\frac{k+1}{2^{j}}\right)= \begin{cases}\frac{\alpha}{2 \tan (\alpha / 2)} \frac{\sin \left(\frac{k+1}{2^{j}} \alpha\right)}{\sin (\alpha)}, & \text { if } k \leq 2^{j}-1, \\ \frac{\alpha}{2 \tan (\alpha / 2)} \frac{\sin \left(\left(2-\frac{k+1}{2^{j}}\right) \alpha\right)}{\sin (\alpha)}, & \text { if } k \geq 2^{j}-1 .\end{cases}
$$

\section{Error analysis and some numerical experiments}

In this section we study the accuracy of the approximation (3.10), starting with the first degree approximation (3.4) and then passing to a general degree approximation. We study the error by considering two steps.

1. The error $e_{1, j}^{U, V}(x):=\phi_{1}^{U, V}(x)-\widetilde{\phi}_{1, j}^{U, V}(x)$ is the standard (piecewise) linear interpolation error with zero values in the points $i / 2^{j}, i=0, \ldots, 2^{j+1}$. It is well known that, if $\varphi \in C^{2}(a, b)$ is the function to be approximated by its linear interpolant at the points $a$ and $b$, denoted by $L_{\{a, b\}} \varphi$, then for every $a<x<b$,

$$
\left|\varphi(x)-L_{\{a, b\}} \varphi(x)\right| \leq \frac{1}{8}(b-a)^{2} \sup _{y \in(a, b)}|\ddot{\varphi}(y)|,
$$

where $\ddot{\varphi}$ is the second derivative of $\varphi$. We consider the intervals $\left[i / 2^{j},(i+1) / 2^{j}\right]$ for $i=$ $0, \ldots, 2^{j+1}-1$. From this, and referring to the notation of $(4.1)$, it follows that $b-a=2^{-j}$, and so $(1 / 8)(b-a)^{2}=2^{-2 j-3}$. 
2. The error $e_{p, j}^{U, V}(x):=\phi_{p}^{U, V}(x)-\widetilde{\phi}_{p, j}^{U, V}(x)$ can be obtained from the error $e_{1, j}^{U, V}(x)$ by convolution. Indeed, we have for $p \geq 2$,

$$
\phi_{p}^{U, V}(x)-\widetilde{\phi}_{p, j}^{U, V}(x)=\left(\left(\phi_{1}^{U, V}-\widetilde{\phi}_{1, j}^{U, V}\right) * \phi_{p-2}\right)(x) .
$$

Let us now work out the details of the error bound.

Lemma 4.1. For every $p \geq 0$ and $x \in \mathbb{R}$, we have

$$
\int_{0}^{2} \phi_{p}(x-y) \mathrm{d} y \leq 2 \phi_{p+1}\left(\frac{p+1}{2}\right)=: I_{p}
$$

and $0 \leq I_{p} \leq 1$

Proof. For $p=0$ it is easy to verify that

$$
\int_{0}^{2} \phi_{0}(x-y) \mathrm{d} y \leq 1=I_{0}
$$

Now we prove (4.3) for $p \geq 1$. From [18] we know that for $t \in[0, p+1]$,

$$
\int_{0}^{t} \phi_{p}(y) \mathrm{d} y=\sum_{k=0}^{p} \phi_{p+1}(t-k)
$$

Hence, for $x \in[2, p+1]$,

$$
\begin{aligned}
\int_{0}^{2} \phi_{p}(x-y) \mathrm{d} y & =\int_{x-2}^{x} \phi_{p}(y) \mathrm{d} y=\sum_{k=0}^{p}\left(\phi_{p+1}(x-k)-\phi_{p+1}(x-k-2)\right) \\
& =\phi_{p+1}(x)+\phi_{p+1}(x-1)-\phi_{p+1}(x-p-1)-\phi_{p+1}(x-p-2) .
\end{aligned}
$$

When taking $x=(p+3) / 2$, we get

$$
\int_{0}^{2} \phi_{p}\left(\frac{p+3}{2}-y\right) \mathrm{d} y=\phi_{p+1}\left(\frac{p+3}{2}\right)+\phi_{p+1}\left(\frac{p+1}{2}\right)-\phi_{p+1}\left(\frac{-p+1}{2}\right)-\phi_{p+1}\left(\frac{-p-1}{2}\right)
$$

and by exploiting the symmetry and local support of the cardinal B-spline, this can be simplified to

$$
\int_{0}^{2} \phi_{p}\left(\frac{p+3}{2}-y\right) \mathrm{d} y=2 \phi_{p+1}\left(\frac{p+1}{2}\right) .
$$

From the symmetric bell-shape of the cardinal B-spline it follows that for any $x \in \mathbb{R}$,

$$
\int_{0}^{2} \phi_{p}(x-y) \mathrm{d} y \leq \int_{0}^{2} \phi_{p}\left(\frac{p+3}{2}-y\right) \mathrm{d} y,
$$

which implies (4.3). Finally, by the positivity and unity of integral of the cardinal B-spline, it is clear from (4.4) that $0 \leq I_{p} \leq 1$.

The quantity $I_{p}$ is visualized in Figure 2 for different $p$, and we see that its value decreases for increasing $p$.

Lemma 4.2. For every $p \geq 2$ and $x \in \mathbb{R}$, we have

$$
\left|\left(e_{1, j}^{U, V} * \phi_{p-2}\right)(x)\right| \leq I_{p-2} \max _{y \in[0,2]}\left|e_{1, j}^{U, V}(y)\right|,
$$

where $I_{q}$ is defined in (4.3). 


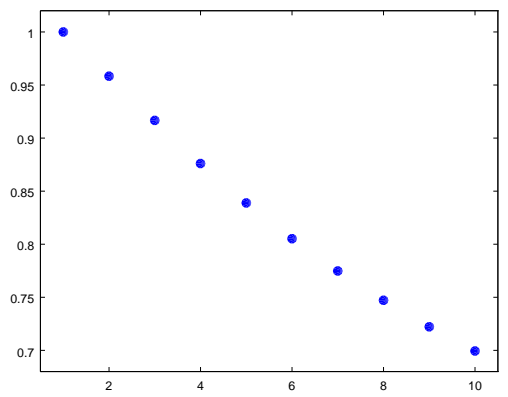

Figure 2: Values of $I_{p}$ defined in (4.3) for $p=1, \ldots, 10$.

Proof. From the definition of convolution and the positivity of cardinal B-splines, we get

$$
\begin{aligned}
\left|\left(e_{1, j}^{U, V} * \phi_{p-2}\right)(x)\right| & =\left|\int_{0}^{2} e_{1, j}^{U, V}(y) \phi_{p-2}(x-y) \mathrm{d} y\right| \leq \int_{0}^{2}\left|e_{1, j}^{U, V}(y)\right| \phi_{p-2}(x-y) \mathrm{d} y \\
& \leq \max _{y \in[0,2]}\left|e_{1, j}^{U, V}(y)\right| \int_{0}^{2} \phi_{p-2}(x-y) \mathrm{d} y,
\end{aligned}
$$

and using Lemma 4.1 completes the proof.

Theorem 4.1. For $p \geq 1$ and $x \in \mathbb{R}$, we have

$$
\left|\phi_{p}^{U, V}(x)-\widetilde{\phi}_{p, j}^{U, V}(x)\right| \leq 2^{-2 j-3} I_{p-2} \sup _{y \in(0,1) \cup(1,2)}\left|\ddot{\phi}_{1}^{U, V}(y)\right|,
$$

where $I_{-1}:=1, I_{q}$ is defined in (4.3) for $q \geq 0$, and $\ddot{\phi}_{1}^{U, V}$ is the second derivative of $\phi_{1}^{U, V}$.

Proof. The result follows immediately from (4.1), (4.2) and (4.5).

We see that the approximation error reduces as $O\left(4^{-j}\right)$ with respect to $j$. To have a precise constant in our error estimate we still need to bound $\left|\ddot{\phi}_{1}^{U, V}(x)\right|$.

Example 4.1. The second derivative of $\phi_{1}^{\mathbb{H}_{\alpha}}(x)$ can be bounded as follows. By the local support and symmetry of $\phi_{1}^{\mathbb{H}_{\alpha}}(x)$ with respect to $x=1$ (see Remark 2.2), we can restrict our analysis to the interval $[0,1]$. We have

$$
\phi_{1}^{\mathbb{H}_{\alpha}}(x)=\frac{\alpha}{2 \tanh (\alpha / 2)} \frac{\sinh (\alpha x)}{\sinh (\alpha)}, \quad 0<x<1,
$$

and differentiating twice results in

$$
\ddot{\phi}_{1}^{\mathbb{H}_{\alpha}}(x)=\frac{\alpha^{3}}{2 \tanh (\alpha / 2)} \frac{\sinh (\alpha x)}{\sinh (\alpha)}, \quad 0<x<1 .
$$

Hence, we conclude

$$
\left|\ddot{\phi}_{1}^{\mathbb{H}_{\alpha}}(x)\right| \leq\left|\frac{\alpha^{3}}{2 \tanh (\alpha / 2)}\right|, \quad x \in \mathbb{R} .
$$

This bound is plotted in Figure 3 (left). 


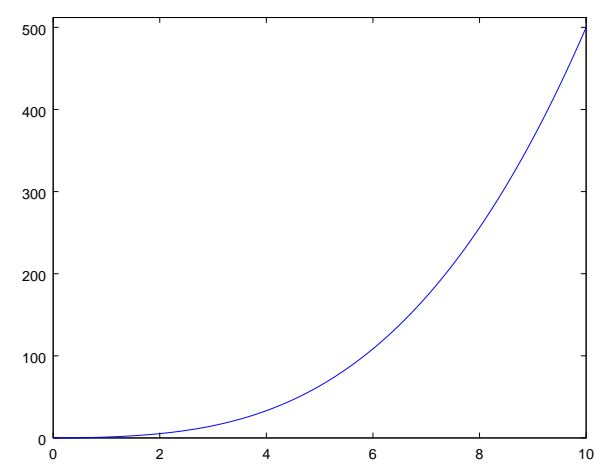

(a) bound for $\left|\ddot{\phi}_{1}^{\mathbb{H}_{\alpha}}(x)\right|$ ranging $\alpha$ in $(0,10)$

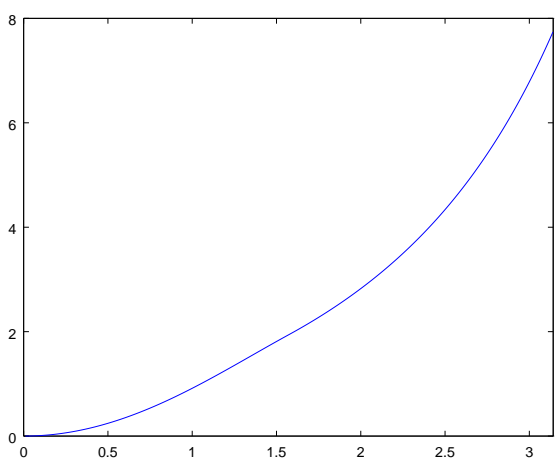

(b) bound for $\left|\ddot{\phi}_{1}^{\mathbb{T}} \alpha(x)\right|$ ranging $\alpha$ in $(0, \pi)$

Figure 3: Graphs of the bounds in (4.7) and (4.8) for $\left|\ddot{\phi}_{1}^{\mathbb{H} \alpha}(x)\right|$ and $\left|\ddot{\phi}_{1}^{\mathbb{T} \alpha}(x)\right|$ with respect to $\alpha$.

Example 4.2. The second derivative of $\phi_{1}^{\mathbb{T}_{\alpha}}(x)$ can be bounded as follows. By the local support and symmetry of $\phi_{1}^{\mathbb{T}_{\alpha}}(x)$ with respect to $x=1$ (see Remark 2.2), we can restrict our analysis to the interval $[0,1]$. We have

$$
\phi_{1}^{\mathbb{T} \alpha}(x)=\frac{\alpha}{2 \tan (\alpha / 2)} \frac{\sin (\alpha x)}{\sin (\alpha)}, \quad 0<x<1,
$$

and differentiating twice results in

$$
\ddot{\phi}_{1}^{\mathbb{T}_{\alpha}}(x)=-\frac{\alpha^{3}}{2 \tan (\alpha / 2)} \frac{\sin (\alpha x)}{\sin (\alpha)}, \quad 0<x<1 .
$$

If $0<\alpha<\frac{\pi}{2}$, then $|\sin (\alpha x)| \leq|\sin (\alpha)|$, whereas if $\frac{\pi}{2} \leq \alpha<\pi$, then $|\sin (\alpha x)| \leq 1$. Hence, we get

$$
\left|\ddot{\phi}_{1}^{\mathbb{T}_{\alpha}}(x)\right| \leq\left\{\begin{array}{ll}
\left|\frac{\alpha^{3}}{2 \tan (\alpha / 2)}\right|, & \text { if } 0<\alpha<\frac{\pi}{2}, \\
\left|\frac{\alpha^{3}}{2 \tan (\alpha / 2) \sin (\alpha)}\right|, & \text { if } \frac{\pi}{2} \leq \alpha<\pi,
\end{array} \quad x \in \mathbb{R} .\right.
$$

This bound is plotted in Figure 3 (right).

We will now check the sharpness of the error bound in (4.6) together with (4.7)-(4.8) in some numerical examples. Let us first consider the hyperbolic cardinal GB-spline case. We start by recalling from Lemma 4.1 that $0 \leq I_{p} \leq 1$. Hence, we know from (4.6) and (4.7) that the approximation error $e_{p, j}^{\mathbb{H}_{\alpha}}(x):=\phi_{p}^{\mathbb{H}_{\alpha}}(x)-\widetilde{\phi}_{p, j}^{\mathbb{H}_{\alpha}}(x)$ is bounded by

$$
E_{j}^{\mathbb{H}_{\alpha}}:=4^{-j-2}\left|\frac{\alpha^{3}}{\tanh (\alpha / 2)}\right| .
$$

This bound is reported in Table 1 for different values of $j$ and $\alpha$. Let $G_{p}$ be the set of points uniformly distributed over the interval $[0, p+1]$ with a stepsize of 0.01 . Table 1 also shows the maximum value of the error $\left|e_{p, j}^{\mathbb{H} \mathbb{H}_{\alpha}}(x)\right|$ sampled over $G_{p}$ for $p=1,2,3$ and for different values of $j$ and $\alpha$. We clearly notice the reduction of the error by a factor $1 / 4$ when moving from $j$ to $j+1$ for fixed $p$ and $\alpha$. Moreover, we observe that the bound (4.9) is a quite sharp approximation of the maximum error value depicted in column $p=1$, especially for higher values of $j$. Finally, we see that the error decreases for increasing $p$. This is expected because we know that $I_{p}$ is decreasing for increasing $p$ (see Figure 2). Some plots of the error are depicted in Figure $4(\mathrm{a}, \mathrm{c})$. 


\begin{tabular}{|c|c|c|c|c|c|}
\hline & & $E_{j}^{\mathrm{H}_{\alpha}}$ & $p=1$ & $p=2$ & $p=3$ \\
\hline \multirow{5}{*}{$\alpha=1$} & $j=1$ & $0.338 \mathrm{e}-1$ & $0.239 \mathrm{e}-1$ & $0.159 \mathrm{e}-1$ & $0.133 \mathrm{e}-1$ \\
\cline { 2 - 6 } & $j=2$ & $0.845 \mathrm{e}-2$ & $0.714 \mathrm{e}-2$ & $0.398 \mathrm{e}-2$ & $0.348 \mathrm{e}-2$ \\
\cline { 2 - 6 } & $j=3$ & $0.212 \mathrm{e}-2$ & $0.194 \mathrm{e}-2$ & $0.996 \mathrm{e}-3$ & $0.879 \mathrm{e}-3$ \\
\cline { 2 - 6 } & $j=4$ & $0.529 \mathrm{e}-3$ & $0.506 \mathrm{e}-3$ & $0.249 \mathrm{e}-3$ & $0.220 \mathrm{e}-3$ \\
\cline { 2 - 6 } & $j=5$ & $0.132 \mathrm{e}-3$ & $0.119 \mathrm{e}-3$ & $0.623 \mathrm{e}-4$ & $0.551 \mathrm{e}-4$ \\
\hline \multirow{5}{*}{$\alpha=10$} & $j=1$ & $0.156 \mathrm{e}+2$ & $0.240 \mathrm{e}+1$ & $0.152 \mathrm{e}+1$ & $0.120 \mathrm{e}+1$ \\
\cline { 2 - 6 } & $j=2$ & $0.391 \mathrm{e}+1$ & $0.132 \mathrm{e}+1$ & $0.470 \mathrm{e}+0$ & $0.409 \mathrm{e}+0$ \\
\cline { 2 - 6 } & $j=3$ & $0.977 \mathrm{e}+0$ & $0.544 \mathrm{e}+0$ & $0.126 \mathrm{e}+0$ & $0.113 \mathrm{e}+0$ \\
\cline { 2 - 6 } & $j=4$ & $0.244 \mathrm{e}+0$ & $0.181 \mathrm{e}+0$ & $0.321 \mathrm{e}-1$ & $0.290 \mathrm{e}-1$ \\
\cline { 2 - 6 } & $j=5$ & $0.610 \mathrm{e}-1$ & $0.475 \mathrm{e}-1$ & $0.807 \mathrm{e}-2$ & $0.731 \mathrm{e}-2$ \\
\hline
\end{tabular}

Table 1: Theoretical bound $E_{j}^{\mathbb{H}_{\alpha}}$ defined in (4.9) and the maximum value of the error $\left|e_{p, j}^{\mathbb{H}_{\alpha}}(x)\right|$ sampled over $G_{p}$.

\begin{tabular}{|c|c|c|c|c|c|}
\hline & & $E_{j}^{\mathbb{I}_{\alpha}}$ & $p=1$ & $p=2$ & $p=3$ \\
\hline \multirow{5}{*}{$\alpha=1$} & $j=1$ & $0.286 \mathrm{e}-1$ & $0.231 \mathrm{e}-1$ & $0.153 \mathrm{e}-1$ & $0.131 \mathrm{e}-1$ \\
\cline { 2 - 6 } & $j=2$ & $0.715 \mathrm{e}-2$ & $0.651 \mathrm{e}-2$ & $0.383 \mathrm{e}-2$ & $0.338 \mathrm{e}-2$ \\
\cline { 2 - 6 } & $j=3$ & $0.179 \mathrm{e}-2$ & $0.171 \mathrm{e}-2$ & $0.956 \mathrm{e}-3$ & $0.851 \mathrm{e}-3$ \\
\cline { 2 - 6 } & $j=4$ & $0.447 \mathrm{e}-3$ & $0.437 \mathrm{e}-3$ & $0.239 \mathrm{e}-3$ & $0.213 \mathrm{e}-3$ \\
\cline { 2 - 6 } & $j=5$ & $0.112 \mathrm{e}-3$ & $0.104 \mathrm{e}-3$ & $0.597 \mathrm{e}-4$ & $0.533 \mathrm{e}-4$ \\
\hline \multirow{5}{*}{$\alpha=3.14$} & $j=1$ & $0.242 \mathrm{e}+0$ & $0.165 \mathrm{e}+0$ & $0.107 \mathrm{e}+0$ & $0.107 \mathrm{e}+0$ \\
\cline { 2 - 6 } & $j=2$ & $0.605 \mathrm{e}-1$ & $0.552 \mathrm{e}-1$ & $0.260 \mathrm{e}-1$ & $0.260 \mathrm{e}-1$ \\
\cline { 2 - 6 } & $j=3$ & $0.151 \mathrm{e}-1$ & $0.148 \mathrm{e}-1$ & $0.644 \mathrm{e}-2$ & $0.644 \mathrm{e}-2$ \\
\cline { 2 - 6 } & $j=4$ & $0.378 \mathrm{e}-2$ & $0.375 \mathrm{e}-2$ & $0.161 \mathrm{e}-2$ & $0.161 \mathrm{e}-2$ \\
\cline { 2 - 6 } & $j=5$ & $0.945 \mathrm{e}-3$ & $0.903 \mathrm{e}-3$ & $0.402 \mathrm{e}-3$ & $0.401 \mathrm{e}-3$ \\
\hline
\end{tabular}

Table 2: Theoretical bound $E_{j}^{\mathbb{T} \alpha}$ defined in (4.10) and the maximum value of the error $\left|e_{p, j}^{\mathbb{T}_{\alpha}}(x)\right|$ sampled over $G_{p}$.

A completely similar behavior is observed in the trigonometric cardinal GB-spline case. We know from (4.6) and (4.8) that the approximation error $e_{p, j}^{\mathbb{T}_{\alpha}}(x):=\phi_{p}^{\mathbb{T}_{\alpha}}(x)-\widetilde{\phi}_{p, j}^{\mathbb{T}_{\alpha}}(x)$ is bounded by

$$
E_{j}^{\mathbb{T}_{\alpha}}:=4^{-j-2} \begin{cases}\left|\frac{\alpha^{3}}{\tan (\alpha / 2)}\right|, & \text { if } 0<\alpha<\frac{\pi}{2}, \\ \left|\frac{\alpha^{3}}{\tan (\alpha / 2) \sin (\alpha)}\right|, & \text { if } \frac{\pi}{2} \leq \alpha<\pi .\end{cases}
$$

This bound is reported in Table 2 together with the maximum value of the error $\left|e_{p, j}^{\mathbb{T}_{\alpha}}(x)\right|$ sampled over $G_{p}$ for $p=1,2,3$ and for different values of $j$ and $\alpha$. Some plots of the error are depicted in Figure $4(\mathrm{~b}, \mathrm{~d})$.

Next, we make a comparison between the approximate values $\widetilde{\phi}_{p, j}^{U, V}(x)$ given in (3.10) and the values obtained by numerical computation of the integral recurrence relation (2.3) using suitable quadrature rules. A careful investigation of the computational cost of the two procedures is quite involved because it deeply depends on their implementations. Therefore, we limit ourselves to estimate the number of evaluations of the function $\phi_{1}^{U, V}$ needed in each of the two approaches. We just focus on the (most common) case where $\phi_{1}^{U, V}$ is symmetric, like for hyperbolic and trigonometric cardinal GB-splines. Taking into account this symmetry, from (3.5) we see that

$$
F_{j}:=2^{j}-1
$$

evaluations of $\phi_{1}^{U, V}$ are required to compute all values $q_{k, j}^{U, V}$ in (3.10)-(3.11). In order to compute numerically the recurrence relation (2.3), we approximate each integral $\int_{0}^{x}\left(\phi_{\ell}^{U, V}(y)-\phi_{\ell}^{U, V}(y-1)\right) \mathrm{d} y$, 


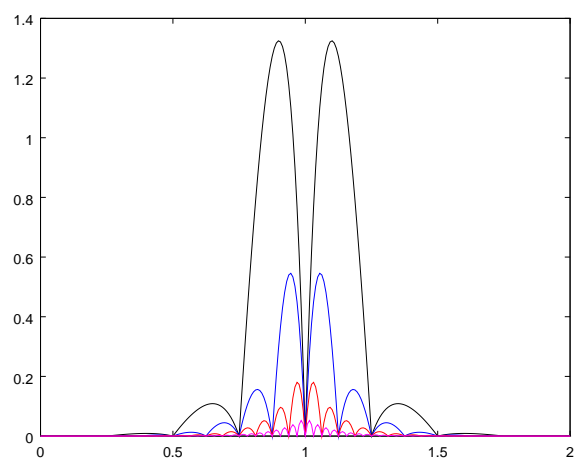

(a) $\mathbb{H}, p=1, \alpha=10$

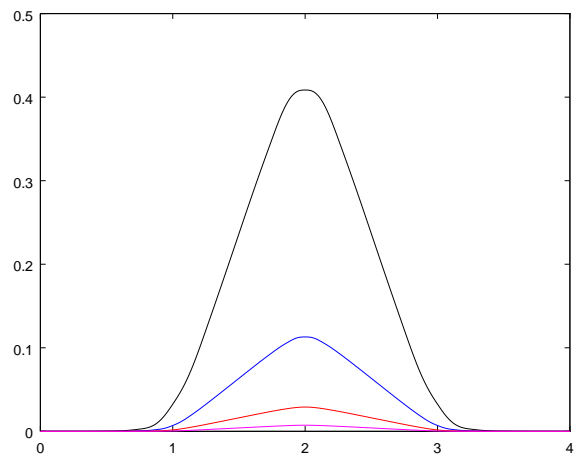

(c) $\mathbb{H}, p=3, \alpha=10$

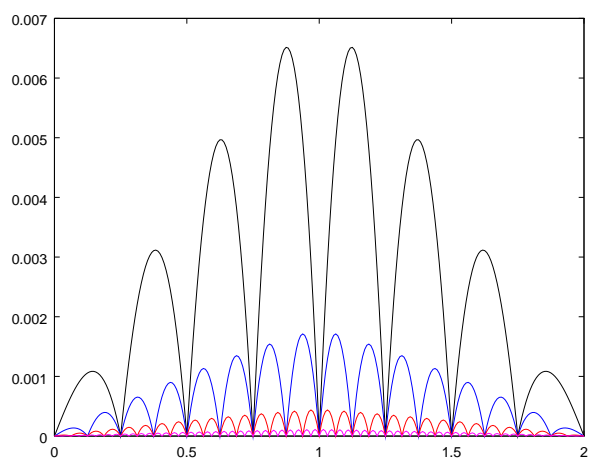

(b) $\mathbb{T}, p=1, \alpha=1$

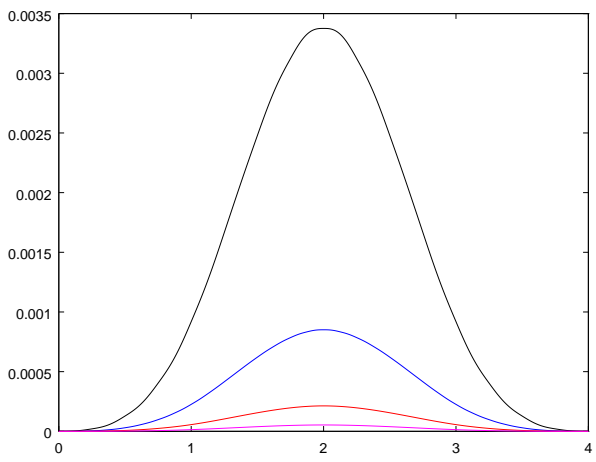

(d) $\mathbb{T}, p=3, \alpha=1$

Figure 4: Plots of $\left|e_{p, j}^{\mathbb{H} \alpha}(x)\right|$ and $\left|e_{p, j}^{\mathbb{T}_{\alpha}}(x)\right|$ for different values of $p$ and $\alpha$. In each plot several refinement levels are considered: $j=2$ (black), $j=3$ (blue), $j=4$ (red), $j=5$ (magenta).

\begin{tabular}{|c|c|c|c|c|c|}
\hline & & $p=2$ & $p=3$ & $p=4$ & $p=5$ \\
\hline \multirow{5}{*}{$\alpha=1$} & $j=6$ & $0.156 \mathrm{e}-4$ & $0.138 \mathrm{e}-4$ & $0.123 \mathrm{e}-4$ & $0.113 \mathrm{e}-4$ \\
\cline { 2 - 6 } & $j=8$ & $0.973 \mathrm{e}-6$ & $0.861 \mathrm{e}-6$ & $0.770 \mathrm{e}-6$ & $0.706 \mathrm{e}-6$ \\
\cline { 2 - 6 } & $m=2$ & $0.128 \mathrm{e}-3$ & $0.365 \mathrm{e}-3$ & $0.445 \mathrm{e}-3$ & $0.122 \mathrm{e}-2$ \\
\cline { 2 - 6 }$\alpha=10$ & $m=3$ & $0.265 \mathrm{e}-6$ & $0.790 \mathrm{e}-6$ & $0.976 \mathrm{e}-6$ & $0.266 \mathrm{e}-5$ \\
\hline \multirow{5}{*}{$\alpha=20$} & $j=6$ & $0.202 \mathrm{e}-2$ & $0.183 \mathrm{e}-2$ & $0.149 \mathrm{e}-2$ & $0.132 \mathrm{e}-2$ \\
\cline { 2 - 6 } & $j=8$ & $0.126 \mathrm{e}-3$ & $0.114 \mathrm{e}-3$ & $0.929 \mathrm{e}-4$ & $0.826 \mathrm{e}-4$ \\
\cline { 2 - 6 } & $m=4$ & $0.470 \mathrm{e}-2$ & $0.350 \mathrm{e}-2$ & $0.311 \mathrm{e}-2$ & $0.305 \mathrm{e}-2$ \\
\cline { 2 - 6 } & $m=5$ & $0.288 \mathrm{e}-3$ & $0.222 \mathrm{e}-3$ & $0.194 \mathrm{e}-3$ & $0.191 \mathrm{e}-3$ \\
\cline { 2 - 6 } & $j=6$ & $0.812 \mathrm{e}-2$ & $0.772 \mathrm{e}-2$ & $0.605 \mathrm{e}-2$ & $0.538 \mathrm{e}-2$ \\
\cline { 2 - 6 } & $j=8$ & $0.509 \mathrm{e}-3$ & $0.483 \mathrm{e}-3$ & $0.379 \mathrm{e}-3$ & $0.337 \mathrm{e}-3$ \\
\cline { 2 - 6 } & $m=5$ & $0.194 \mathrm{e}-1$ & $0.134 \mathrm{e}-1$ & $0.128 \mathrm{e}-1$ & $0.122 \mathrm{e}-1$ \\
\hline & $m=6$ & $0.278 \mathrm{e}-2$ & $0.196 \mathrm{e}-2$ & $0.186 \mathrm{e}-2$ & $0.179 \mathrm{e}-2$ \\
\hline
\end{tabular}

Table 3: Maximum value of the error $\left|e_{p, j}^{\mathbb{H}_{\alpha}}(x)\right|$ obtained by approximation strategy (3.10) with different $j$, and maximum value of the error obtained by approximating the integral recurrence relation (2.3) for $\phi_{p}^{\mathbb{H} \alpha}(x)$ by means of a composite Gauss-Legendre quadrature rule with different $m_{\ell}=m$. Both the errors are sampled over $G_{p}$.

$\ell=1, \ldots, p-1$, by a composite Gauss-Legendre quadrature rule based on $m_{\ell}$ quadrature points in each knot (sub)interval. Due to the recursive structure of (2.3), the approximation of $\phi_{p}^{U, V}(x)$ 


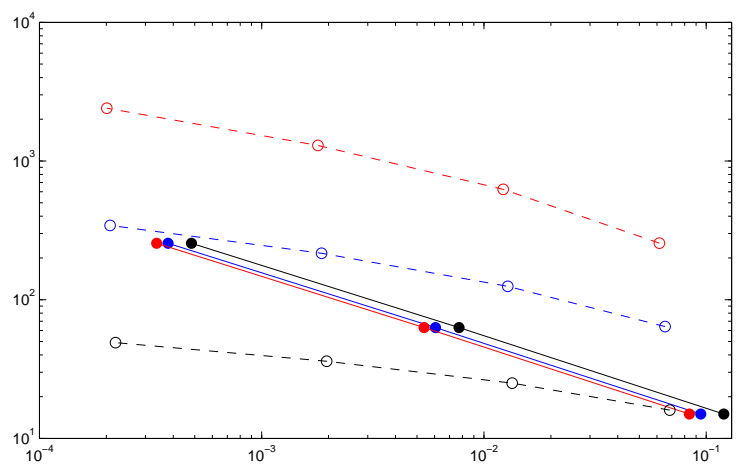

Figure 5: Values of $F_{j}=2^{j}-1$ (for $j=4,6,8$, indicated by filled bullets) and $M_{p}=(m)^{p-1}$ (for $m=4,5,6,7$, indicated by open bullets) plotted against the corresponding maximum error values obtained when approximating $\phi_{p}^{\mathbb{H}_{20}}(x): p=3$ (black), $p=4$ (blue) and $p=5$ (red). See also data in Table 3.

requires $C(x) M_{p}$ evaluations of $\phi_{1}^{U, V}$ where $C(x) \geq 1$ depends on the knot interval containing $x$ and

$$
M_{p}:=m_{p-1} m_{p-2} \cdots m_{1} .
$$

The value $j$, necessary to reach a given accuracy when using (3.10), can be easily determined by the error bound in Theorem 4.1 (and also (4.9) and (4.10) for the hyperbolic and trigonometric case, respectively). On the contrary, an accurate selection of $m_{\ell}$ is an arduous task; $m_{\ell}$ should be large enough to integrate exactly polynomials of degree $\ell$ and approximately well the functions $U^{(p-\ell)}, V^{(p-\ell)}$.

Table 3 reports the maximum errors obtained by the two approaches when approximating $\phi_{p}^{\mathbb{H}_{\alpha}}(x)$ over the grid $G_{p}$ for different values of the parameter $\alpha$ and the degree $p$; we also vary the approximation level $j$ and the number of the quadrature points $m_{\ell}=m$. The range of values for $\alpha$ and $p$ considered in the test are of practical interest in applications. In particular, the values $\alpha=20$ and $p=4$ are those used in [15, Section 4.2.2], considering a proper scaling of the variable $x$. Figure 5 depicts in logarithmic scale the cost values $F_{j}=2^{j}-1$ (for $j=4,6,8$ ) and the lower bound $M_{p}=(m)^{p-1}$ (for $\left.m=4,5,6,7\right)$ versus the corresponding maximum error values obtained when approximating $\phi_{p}^{\mathbb{H}_{\alpha}}(x)$ with $\alpha=20$ and $p=3,4,5$.

\section{Conclusions}

GB-splines - the B-spline-like basis for generalized splines - are usually defined by means of an integral recurrence relation which makes their evaluation quite cumbersome and computationally expensive. This inherent complexity often results in a reluctant attitude to use GB-splines in practical applications, despite their interesting analytical and geometrical properties. To circumvent this barrier, we have proposed an approximation strategy for the evaluation of cardinal GB-splines of arbitrary degree $p$. The approach is based on convolution of cardinal B-splines and its accuracy can be predetermined according to the application we need to deal with.

The proposed approximation method deeply exploits the Fourier properties of both polynomial and generalized cardinal B-splines. In such a manner we can avoid evaluation of integrals and related numerical quadrature. The approximating function is expressed as a linear combination of scaled and dilated versions of (polynomial) cardinal B-splines of degree $p$, whose coefficients can be efficiently computed via discrete convolution. Moreover, in case a better accuracy is needed, the proposed method allows us to construct a new approximation from the previous approximation by reusing all the computations previously made. 


\section{Acknowledgements}

This work was partially supported by INdAM-GNCS Gruppo Nazionale per il Calcolo Scientifico and by the MIUR 'Futuro in Ricerca 2013' Programme through the project DREAMS.

\section{References}

[1] C. de Boor. A Practical Guide to Splines, Revised edition. Springer (2001).

[2] T. Bosner. Knot insertion algorithms for Chebyshev splines. PhD thesis, Dept. of Mathematics, University of Zagreb (2006).

[3] C. Bracco and D. Cho. Generalized T-splines and VMCR T-meshes. Comput. Methods Appl. Mech. Engrg. 280 (2014), 176-196.

[4] C. Bracco, T. Lyche, C. Manni, F. Roman, and H. Speleers. Generalized spline spaces over Tmeshes: Dimension formula and locally refined generalized B-splines. Appl. Math. Comput. 272 (2016), 187-198.

[5] C. Bracco, T. Lyche, C. Manni, F. Roman, and H. Speleers. On the dimension of Tchebycheffian spline spaces over planar T-meshes. Comput. Aided Geom. Design 45 (2016), 151-173.

[6] C. Bracco and F. Roman. Spaces of generalized splines over T-meshes. J. Comput. Appl. Math. 294 (2016), 102-123.

[7] C.K. Chui. An Introduction to Wavelets. Academic Press (1992).

[8] P. Costantini, T. Lyche, and C. Manni. On a class of weak Tchebycheff systems. Numer. Math. 101 (2005), 333-354.

[9] P. Costantini, C. Manni, F. Pelosi, and M.L. Sampoli. Quasi-interpolation in isogeometric analysis based on generalized B-splines. Comput. Aided Geom. Design 27 (2010), 656-668.

[10] C. Garoni, C. Manni, F. Pelosi, S. Serra-Capizzano, and H. Speleers. On the spectrum of stiffness matrices arising from isogeometric analysis. Numer. Math. 127 (2014), 751-799.

[11] B.I. Kvasov and P. Sattayatham. GB-splines of arbitrary order. J. Comput. Appl. Math. 104 (1999), 63-88.

[12] T. Lyche. A recurrence relation for Chebyshevian B-splines. Constr. Approx. 1 (1985), 155-173.

[13] E. Mainar, J.M. Peña, and J. Sánchez-Reyes. Shape preserving alternatives to the rational Bézier model. Comput. Aided Geom. Design 18 (2001), 37-60.

[14] C. Manni, F. Pelosi, and M.L. Sampoli. Generalized B-splines as a tool in isogeometric analysis. Comput. Methods Appl. Mech. Engrg. 200 (2011), 867-881.

[15] C. Manni, A. Reali, and H. Speleers. Isogeometric collocation methods with generalized B-splines. Comput. Math. Appl. 70 (2015), 1659-1675.

[16] M.L. Mazure. How to build all Chebyshevian spline spaces good for geometric design? Numer. Math. 119 (2011), 517-556.

[17] F. Roman, C. Manni, and H. Speleers. Spectral analysis of matrices in Galerkin methods based on generalized B-splines with high smoothness. Numer. Math. (to appear). DOI: 10.1007/s00211016-0796-z

[18] L.L. Schumaker. Spline Functions: Basic Theory, Third edition. Cambridge U.P. (2007).

[19] G. Wang and M. Fang. Unified and extended form of three types of splines. J. Comput. Appl. Math. 216 (2008), 498-508.

[20] H.S. Wilf. Generatingfunctionology. A.K. Peters (2006). 